\title{
Generalized fluctuation-dissipation relation and effective temperature in off-equilibrium colloids
}

\author{
Claudio Maggi, ${ }^{1, *}$ Roberto Di Leonardo, ${ }^{2}$ Jeppe C. Dyre, ${ }^{1}$ and Giancarlo Ruocco ${ }^{3,2}$ \\ ${ }^{1}$ DNRF Centre “Glass and Time," IMFUFA, Department of Sciences, Roskilde University, P.O. Box 260, DK-4000, Roskilde, Denmark \\ ${ }^{2}$ Research Center "Soft," INFM-CNR, clo Department of Physics, "Sapienza” University of Rome, Italy \\ ${ }^{3}$ Department of Physics, "Sapienza" University of Rome, Piazzale Aldo Moro 5, I-00185 Rome, Italy \\ (Received 27 November 2009; revised manuscript received 22 January 2010; published 9 March 2010)
}

\begin{abstract}
The fluctuation-dissipation theorem (FDT), a fundamental result of equilibrium statistical physics, can be violated when a system is taken out of equilibrium. A generalization of FDT has been theoretically proposed for out-of-equilibrium systems; the kinetic temperature entering the fluctuation-dissipation relation (FDR) is substituted by a time-scale-dependent effective temperature. We combine the measurements of the correlation function of the rotational dynamics of colloidal particles obtained via dynamic light scattering with those of the birefringence response to study the generalized FDR in an off-equilibrium Laponite suspension undergoing aging. (i) We find that the FDT is strongly violated in the early stage of the aging process and is gradually recovered as the aging time increases and (ii) we determine the aging-time evolution of the effective temperature, comparing our results with those of previous experiments.
\end{abstract}

DOI: 10.1103/PhysRevB.81.104201

PACS number(s): 05.40.-a, 05.45.-a, 05.70.Ln, 47.50.-d

\section{INTRODUCTION}

The study of the dynamics in nonequilibrium systems is an intriguing and fascinating area of modern physics. There is an obvious interest in the off-equilibrium regime as condensed matter is often found far from ideal equilibrium condition where "things keep happening on all time scales." ${ }^{1} \mathrm{~A}$ particular class of systems where the out-of-equilibrium status occurs naturally are the so-called glass-forming systems. These systems include, for example, disordered spin systems close to the spin-glass transition, supercooled molecular liquids, and jamming colloidal solutions. In general, the dynamics of these systems is extremely sensitive to change in external parameters so that, when they are a little cooled or densified, the time required to reestablish equilibrium can grow enormously or become infinite (the system never reaches the final equilibrium state). In these situations, an off-equilibrium regime is entered. In this, the average quantities becomes time dependent and the correlation and response functions depend on two times; in this regime the system is aging.

Correlation functions and response functions are the basic quantities through which one probes the dynamics of a system in many-body statistical physics. These functions are closely related in equilibrium by the fluctuation-dissipation theorem (FDT). The FDT (Ref. 2) establishes a relationship between the correlation function $C_{A B}(t)=\langle A(0) B(t)\rangle$ of the spontaneous equilibrium fluctuations of the dynamic variables $A$ and $B$ and the response function $\chi_{A B}(t)=\langle A(t)\rangle / h$, describing the change in the average value of $A$ due to an infinitesimal external field $h$ that is coupled to the variable $B$ in the perturbation Hamiltonian,

$$
\chi_{A B}(t)=\beta\left[C_{A B}(0)-C_{A B}(t)\right] .
$$

Here $\beta=1 / k_{B} T$, where $T$ is the kinetic temperature of the system and $A$ and $B$ are variables that have zero mean in the unperturbed case. In this formulation of the FDT, the field $h$ introduces a contribution $\delta H=-h B$ to the system Hamiltonian, which is switched on instantaneously at $t=0$ and kept constant for $t>0$ as a Heaviside step function. In the following, we will refer to autocorrelation function $(B=A)$ and drop the label $A A$ in $C_{A A}(t)$ and $\chi_{A A}(t)$.

Out of equilibrium, the system is nonstationary and timetranslational invariance is lost. The correlation and the response become two-times quantities depending also on the aging time $t_{w}: C=C\left(t_{w}, t_{w}+t\right), \chi=\chi\left(t_{w}, t_{w}+t\right)$, and the FDT [Eq. (1)] is not expected to hold. The importance of extending the theorem to the nonequilibrium regime has led to the generalized fluctuation-dissipation relation (GFDR) ${ }^{3-5}$

The generalization of the FDT proposed by Kurchan and Cugliandolo $^{6,7}$ in the early 1990 s can be expressed as follows:

$$
\chi\left(t_{w}, t_{w}+t\right)=\beta \int_{t_{w}+t}^{t_{w}} d s X\left(t_{w}, s\right) \partial_{s} C\left(t_{w}, s\right),
$$

where for short times $t$ (i.e., $t / t_{w} \ll 1$ ), this equation reduces to the FDT [Eq. (1)] and the system is said to be in a quasiequilibrium state; for intermediate $t\left(t / t_{w} \simeq 1\right)$, the violation function $X\left(t_{w}, t\right)$ quantifies the deviation of the GFDR from the FDT; finally, when $t / t_{w} \gg 1$, the function $X$ depends on $t_{w}$ and $s$ only through the function $C$. The violation function can also be interpreted ${ }^{8,9}$ in terms of an effective temperature $T_{e f f}=T / X$. The latter has complicated behavior for intermediate $t$ values, but in the long- $t$ regime it assumes a value that depends only on the waiting time $t_{w}$ and one can think of it as "waiting-time-dependent effective temperature."

The GFDR in out-of-equilibrium systems has been studied theoretically and through computer simulations in spin glasses ${ }^{10,11}$ and in models for glassy dynamics. ${ }^{12}$ Later, offequilibrium molecular-dynamics simulations have given the possibility to perform the same analysis on structural glasses. ${ }^{13-15}$ Recently, the generalized relation has also been investigated in more exotic systems, such as in the simulations for a glassy protein,${ }^{16}$ in self-assembling processes of viral capsids formation and of sticky disks crystallization, ${ }^{17}$ and in active systems composed of self-propelled particles. ${ }^{18}$ 
On the other hand, few experiments have studied the GFDR. ${ }^{19-24}$ Experiments in structural glasses are difficult because it is necessary to simultaneously measure a correlation function of a given variable and the associated response function, and all this in a systems that is instantaneously brought out of equilibrium. These difficulties are relaxed in the case of colloidal glasses (or gels or jams) because the associated time scales are much longer and fall easily in the experimentally accessible window.

In this paper, we report an experimental investigation of the generalized FDR in a colloidal systems composed of a water suspension of clay platelets (Laponite), which is off-equilibrium when it ages toward the final arrested state (gel or glass, depending on the clay concentration ${ }^{25}$ ).

We emphasize that the FDT violation and the effective temperature in these colloidal solution are object of intense debate since the results published so far are controversial (see, for example, Refs. 21-24 and 26). Indeed, previous works on off-equilibrium Laponite suspensions have reported contradicting results in studying the GFDR. Dielectric spectroscopy combined with spontaneous polarization-noise measurements have revealed very high effective temperatures characterizing the low-frequency part of the spectra. This effective temperature was found to decrease with the aging time.

Later a new series of experiments employed microrheology to measure the effective temperature of these colloidal systems. These experiments studied the Brownian motion of probe particles added to the Laponite solution. The results of these experiments are rather contradicting. Abou et al. ${ }^{26} \mathrm{ob}-$ served an effective temperature evolving nonmonotonically as a function of the aging time. $T_{\text {eff }}$ was found to increase from the bath temperature $T$, reach a maximum and return to $T$ for long times. In contrast, the results of Ref. 22 by Jabbari-Farouji et al. indicated that no significant deviation from the FDT takes place over a wide frequency range. These conclusions were later confirmed by Jop et al. ${ }^{24} \mathrm{Fi}-$ nally, the experiments of Greinert et al. ${ }^{23}$ measured an effective temperature increasing with aging time.

We want to stress that none of these works studied directly the degrees of freedom of the colloidal Laponite disks. Indeed, the dielectric techniques are mostly sensitive to the strong polarization signal from the solvent molecules and to the effect of ionic conduction at low frequencies. In the microrheological measurements, some large trapped probe particles (typically $\sim 1 \mu \mathrm{m}$ diameter) are used to study the offequilibrium dynamics of the system. In contrast, our experiment is selective in measuring the dynamics of specific degrees of freedom of the Laponite disks (as will be clear in the following). Our correlation and response optical measurements (Secs. III and IV) are sensitive only to the (rotational) motion of the colloids that we are interested in. Moreover, we emphasize that, in principle, is also possible that different observables give different values of the FDT violation and therefore of the effective temperature. Our experiment is a fully time-resolved experiment.

\section{OFF-EQUILIBRIUM SAMPLE}

A solution, prepared by stirring the Laponite powder with water, evolves toward an arrested state on a time scale that spans the ranges hours to months when kept at room temperature and pressure. Even low-concentration aqueous solutions of this colloid, as the one used in our experiment $(\sim 1 \%$ Laponite weight fraction), show strong aging of their lightscattering correlation function. ${ }^{27}$ Due to the long (with respect to the experimental time scale and to the decorrelation time) aging process of the systems, one can approximate the different measure of fluctuations and response as obtained in a sequence of steady out of equilibrium states.

The anisotropic shape of the clay disk makes it possible to study its reorientation dynamics via the response and the correlation function. Laponite particles dissolved in water have the form of flat cylinders with a diameter of $25 \mathrm{~nm}$ and thickness of about $1 \mathrm{~nm}$. Laponite colloidal particles are good scatterers of visible light and this allow us to rapidly measure the autocorrelation function of the scattered field.

In this work we study the reorientational dynamics of the asymmetric clay platelets, looking at the orientational correlation functions via depolarized dynamic light scattering and at the corresponding response function via the electric field induced birefringence. Measuring both $C=C\left(t_{w}, t_{w}+t\right)$ and $\chi=\chi\left(t_{w}, t_{w}+t\right)$ at different waiting time during the (days long) aging process, we find that FDT is strongly violated in the early stage of the aging process and is gradually recovered as the aging time increases. Moreover, from the parametric $C-\chi$ plot (the so-called "FDT plot"), we determine the effective temperature and follow its evolution from the high values $\left(T_{e f f} / T \approx 5\right)$ pertaining to young systems toward the equilibrium $\left(T_{e f f} / T=1\right)$ attained at long waiting time. Our findings confirm the generalization of the FDT to offequilibrium systems proposed by Cugliandolo and Kurchan 15 years ago.

\section{CORRELATION FUNCTION}

In a dynamic light-scattering experiment one measures the correlation function of the optical field scattered by the sample. The scattered field can be directly related to the translational and rotational motion of the anisotropic colloids suspended in the solvent. ${ }^{28}$ The colloids' rotations are related to the second-rank tensor of the optical susceptibility. Specifically, in the $\mathrm{VH}$ (depolarized) scattering geometry one measures the autocorrelation function of a variable that depends on the platelet's orientation,

$$
A(t)=\sum_{i} P_{2}\left\{\cos \left[\theta_{i}(t)\right]\right\}
$$

where $P_{2}(x)=\left(3 x^{2}-1\right) / 2$ is the second-order Legendre polynomial, $\theta_{i}$ is the angle formed by the symmetry axes of the $i$ th particle with the polarization vector of the incident field, and the sum is extended over the particles contained in the scattering volume. ${ }^{28}$ This results holds exactly only if the time scale of the rotational dynamics is much faster than the translational one. This assumption was confirmed by comparing the $\mathrm{VV}$ (polarized) and $\mathrm{VH}$ (depolarized) photoncorrelation spectroscopy (PCS) at different waiting times and clay concentration. ${ }^{29}$ The autocorrelation function of $A$ was measured using the VH geometry via PCS. Several autocorrelation functions have been measured during the aging pro- 
cess of the sample with a time resolution $(1 \mu \mathrm{s})$ dictated by the detector (photomultiplier) response. Note that the time is set to zero at the instant at which we stop stirring the colloidal suspension.

\section{RESPONSE FUNCTION}

If one applies an external field that tends to align the particles, the system due to the anisotropic platelet's polarizability becomes birefringent. ${ }^{30,31}$ If the aligning field is a dc (or low frequency) electric field (Kerr effect), the degree of rotation of a linearly polarized laser beam is proportional to the square of the electric field via a coefficient that is proportional to $A$ [Eq. (3)]. Therefore, the (time-dependent) Kerr response to the switch on of an electric field is proportional to the desired response function (i.e., the response conjugated to the correlation function measured in depolarized PCS). For selected values of the waiting times, the timeresolved response functions and the corresponding correlation functions were measured during the aging process of the Laponite solution. The length of the electric pulses produced sets the dynamic window of our experiment to about $1 \mathrm{~ms}$.

Note that the relaxation time $\tau$ of these functions is always much smaller than the typical waiting time $\left(\tau \ll t_{w}\right)$. This means that the time-resolved correlation and response are well-defined quantities although the system is aging. In addition, if any FDT violation can be detected, we expect to find it on a time scale comparable to the relaxation time $(t / \tau \geq 1)$ (Fig. 1).

\section{RESULTS AND DISCUSSION}

Examples of the measured quantities are shown in the left panel of Fig. 2. The correlation function and the response function are reported as functions of $t$ for different aging times $t_{w} \cdot{ }^{32}$ For short $t$, the FDT holds while we can see a clear deviation from the FDT for long $t$ where $\chi / \beta$ does not overlap with $1-C[C(t)$ is normalized to $C(0)=1]$. When $\chi\left(t_{w}, t_{w}+t\right) / \beta$ is parametrically plotted against $C\left(t_{w}, t_{w}+t\right)$ using $t$ as a parameter (FDT plot), the departure from the $1-C$ line becomes evident (see the right panel of Fig. 2). The deviation from the behavior expected from the FDT reduces its importance as $t_{w}$ grows, and the time where $T \chi$ and $C$ detaches from each other moves to longer $t$ [see also Fig. 3(a) where the interested region of the FDT plot has been expanded]. In order to quantify this deviation, we introduced a linear fit to the longer-time points in the FDT plot. The slopes $(m)$ of these lines are a measure of the effective temperature, $1 / m=T_{e f f} / T$.

The $t_{w}$ dependence of $T_{\text {eff }}$ is reported in Fig. 3(b). $T_{\text {eff }}$ decreases as $t_{w}$ increases. The linear fit to the long- $t$ region of the FDT plot also defines a characteristic value of the correlation $C$ where the FDT breaks down, the so-called EdwardsAnderson value $q$; this quantity is reported as a function of $t_{w}$ in Fig. 3(c). Finally, the quantity $q$, defined via $C\left(t_{0}\right)=q$, identifies a characteristic time $t_{0}$ that mark the "starting time" of the violation. $t_{o}$ is found to move to higher values as the aging time increases [Fig. 3(d)]. It is interesting to compare $t_{0}$ to the relaxation times $\tau$ of the response and the

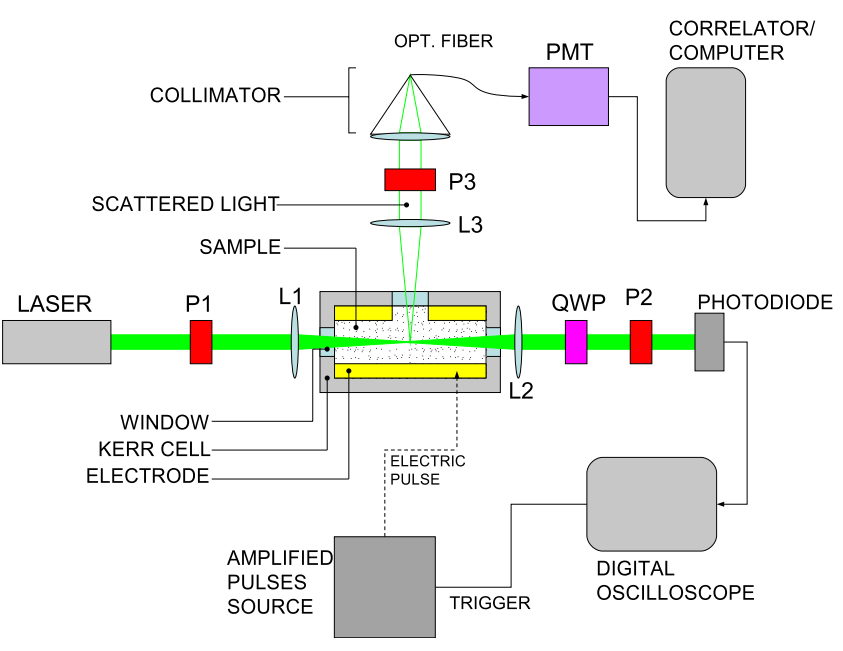

FIG. 1. (Color online) Sketch of the experimental setup (see Ref. 29 for more details). The laser's radiation $(\lambda=532 \mathrm{~nm})$ is polarized by the polarizer P1 and focused by the lens L1 at the center of the cell containing the sample. The scattered light is collected by the lens L3 and selected by the polarizer P3 (orthogonal to P1). A photomultiplier tube (PMT) detects the scattered photons. When no electric pulse is applied to the cell, the output of the PMT is acquired by a computer equipped with a custom digital correlator, this measures and stores the correlation function. The Kerr cell containing the sample is provided by two electrodes connected to a source of amplified electric pulses. The forward-scattered light (rotated by the electrically stimulated sample) is collected by the lens L2 and passes through a quarter-wave plate and the polarizer P2 (orthogonal to P1). The transmitted light is detected by a photodiode connected to a digital oscilloscope. This is triggered to the source of electric pulse measuring and storing the Kerr-response function.

correlation. We find $\tau$ fitting the correlation and the response with stretched exponentials of the form $\exp \left[-(t / \tau)^{\beta}\right]$ and $\left(1-a \exp \left[-(t / \tau)^{\beta}\right]\right)$, respectively. The response ages faster than the correlation, almost reaching the same relaxation time for the longest $t_{w}$.

It is important to emphasize that in all models investigated so far for studying the GFDR, the relaxation time grows roughly as the waiting time, $\tau \sim t_{w}$. The aging process that we study experimentally here does not obey this simple scaling. Indeed, the off-equilibrium (aging time-dependent) relaxation time measured is several orders of magnitude shorter than the typical values of $t_{w}$. Nevertheless, our results show that the different time scales (the one where the dynamics is at the equilibrium and the one where that is out of the equilibrium) are set by the aging time $t_{w}$. Indeed, the $\tau\left(t_{w}\right)$ that grows as the sample ages marks the transition from the regime where the FDT holds to another where the FDT is violated.

Finally, we mention that these results show a qualitative behavior that is similar to the one found in dielectric measurements (performed in the frequency domain). $T_{e f f}$ is equal to the bath temperature for short times (at high frequency) while it is higher than $T$ for long times (at low frequency). In addition, the time scale for the deviation of $T_{\text {eff }}$ from $T$ increases as $t_{w}$ increases (the frequency at which the FDT is violated shifts to lower frequencies).

Although these similarities are intriguing, we stress that some profound differences are also found. First, the relevant 



FIG. 2. (Color online) (Left) Normalized time-correlation function $(\bigcirc)$ and response function $(\square)$ measured at different aging times (from top to bottom: $t_{w}=90,125,225$, and $1200 \mathrm{~min}$ ), the solid line represents $1-C$. Note that $1-C$ deviates from $\chi / \beta$ when the FDT is violated. The importance of this deviation reduces as $t_{w}$ increases. (Right) Response function vs correlation function measured at different aging times $(\bigcirc)$. The black line represents the expectation of the FDT while the color lines represent the linear fits to the points in the off-equilibrium section of the FDT plot. It can be appreciated how these points corresponding to long-time gradually approach the FDT as the aging time increases, for the longest waiting time the fitting line overlaps almost perfectly with the prediction of the FDT (see Fig. 3 for a comparison of the FDT plots at different aging times).

time scales detected are very different. In our experiment, the deviation from the FDT is found at times between 0.1 and 1 ms (i.e., at frequencies between 10 and $1 \mathrm{kHz}$ ). In the studies based on the dielectric response, the violation of the FDT was detected at much lower frequencies (between 10 and 1 $\mathrm{Hz}$ ). Second, the effective temperature found from dielectric measurements was extremely high (orders of magnitude higher than the bath temperature) while we measure a $T_{\text {eff }}$ at most a factor 5 higher than $T$. Note that our measurements
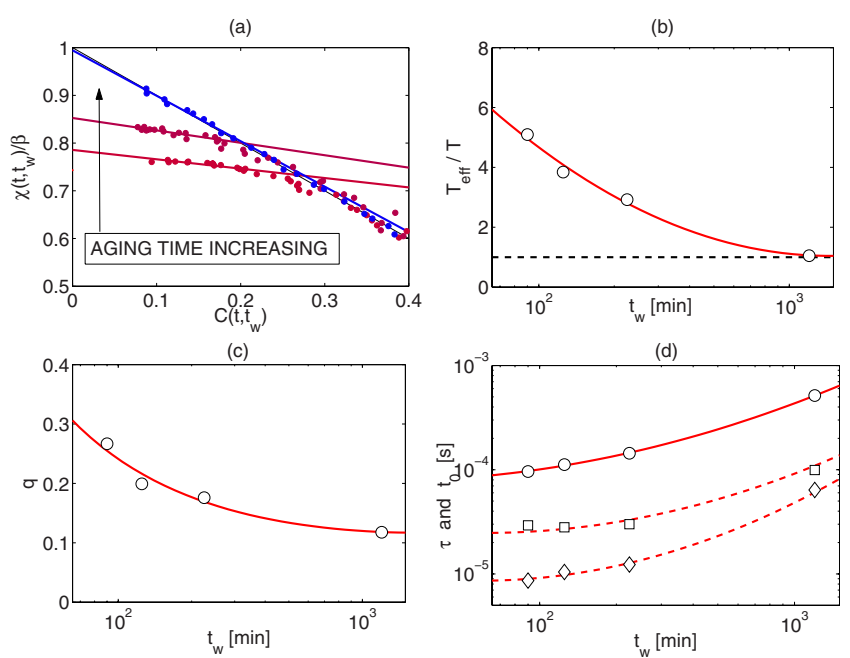

FIG. 3. (Color online) (a) The interesting region of the FDT plots for different aging times: $t_{w}=90,125$, and $1200 \mathrm{~min}$ (cf. Fig. 2, right panel). (b) The inverse slope of the long-time points of the FDT plot (see Fig. 2) as a function of the aging time (the full line is a guide for the eye). This parameter can be interpreted as an effective temperature and it is found to reduce to the bath temperature as the aging time increases. (c) Waiting-time dependence of the Edwards-Anderson parameter, the characteristic value of the correlation function at which the FDT breaks down. (d) Evolution with $t_{w}$ of the characteristic time at which the FDT is violated $(\bigcirc)$, together with the relaxation time of the correlation function $(\square)$ and of the response $(\diamond)$. The characteristic time for the FDT violation $t_{o}$ increases as $t_{w}$ grows followed by the two relaxation times.

(as the dielectric measurement) give a different result with respect to the various findings obtained via the microrheological measurement mentioned in Sec. I.

In conclusion, by measuring the autocorrelation function of a given variable and the response function of the same quantity in an off-equilibrium (aging) colloidal suspension in the route to the arrested state, we have investigated the generalized fluctuation-dissipation relation. The GFDR applies to the present experiment in the following sense: for shorttime scales the FDT is fulfilled, while for long times a clear violation is observed. The characteristic time at which the violation is seen, is always slightly above the aging-timedependent relaxation time of the measured response and correlation function. Moreover, we observe that the deviation from the standard FDT reduces gradually as the aging time increases and the arrested phase is approached. *cmaggi@ruc.dk

${ }^{1}$ R. P. Feynman, Statistical Mechanics: A Set of Lectures, edited by Jacob Shaham (Academic, New York, 1972).

${ }^{2}$ See, for example, the textbooks: J.-P. Hansen and I. R. McDonald, Theory of Simple Liquids, 3rd ed. (Academic, New York, 2006), Chap. 7; L. D. Landau and E. M. Lifshitz, Statistical Physics (Butterworth-Heinemann, Oxford, 1980), Vol. 5;
D. Chandler, Introduction to Modern Statistical Mechanics (Oxford University Press, New York, 1987).

${ }^{3}$ A. Crisanti and R. Ritort, J. Phys. A 36, R181 (2003).

${ }^{4}$ U. M. Marconi, A. Puglisi, L. Rondoni, and A. Vulpiani, Phys. Rep. 461, 111 (2008)

${ }^{5}$ L. F. Cugliandolo, Slow Relaxations and Nonequilibrium Dynamics in Condensed Matter (Springer, Berlin/Heidelberg, 
2004).

${ }^{6}$ L. F. Cugliandolo and J. Kurchan, Phys. Rev. Lett. 71, 173 (1993).

${ }^{7}$ L. F. Cugliandolo and J. Kurchan, J. Phys. A 27, 5749 (1994).

${ }^{8}$ L. F. Cugliandolo, J. Kurchan, and L. Peliti, Phys. Rev. E 55, 3898 (1997).

${ }^{9}$ A. Garriga and F. Ritort, Eur. Phys. J. B 20, 105 (2001); 21, 115 (2001).

${ }^{10}$ M. Mézard, G. Parisi, and M. A. Virasoro, Spin Glass Theory and Beyond (World Scientific, Singapore, 1987).

${ }^{11}$ Spin Glasses and Random Fields, edited by A. P. Young(World Scientific, Singapore, 2000).

${ }^{12}$ G. Diezemann, J. Chem. Phys. 123, 204510 (2005).

${ }^{13}$ G. Parisi, Phys. Rev. Lett. 79, 3660 (1997).

${ }^{14}$ J. L. Barrat and W. Kob, Europhys. Lett. 46, 637 (1999).

${ }^{15}$ R. Di Leonardo, L. Angelani, G. Parisi, and G. Ruocco, Phys. Rev. Lett. 84, 6054 (2000).

${ }^{16}$ K. Hayashi and M. Takano, Biophys. J. 93, 895 (2007).

${ }^{17}$ R. L. Jack, M. F. Hagan, and D. Chandler, Phys. Rev. E 76, 021119 (2007).

${ }^{18}$ D. Loi, S. Mossa, and L. F. Cugliandolo, Phys. Rev. E 77, 051111 (2008).

${ }^{19}$ D. Herisson and M. Ocio, Phys. Rev. Lett. 88, 257202 (2002).

${ }^{20}$ T. S. Grigera and N. E. Israeloff, Phys. Rev. Lett. 83, 5038 (1999).

${ }^{21}$ L. Bellon, S. Ciliberto, and C. Laroche, Europhys. Lett. 53, 511 (2001).
${ }^{22}$ S. Jabbari-Farouji, D. Mizuno, M. Atakhorrami, F. C. MacKintosh, C. F. Schmidt, E. Eiser, G. H. Wegdam, and D. Bonn, Phys. Rev. Lett. 98, 108302 (2007)

${ }^{23}$ N. Greinert, T. Wood, and P. Bartlett, Phys. Rev. Lett. 97, 265702 (2006).

${ }^{24}$ P. Jop, A. Petrosyan, and S. Ciliberto, Philos. Mag. 88, 4205 (2008).

${ }^{25}$ B. Ruzicka, L. Zulian, R. Angelini, M. Sztucki, A. Moussaid, and G. Ruocco, Phys. Rev. E 77, 020402(R) (2008).

${ }^{26}$ B. Abou and F. Gallet, Phys. Rev. Lett. 93, 160603 (2004).

${ }^{27}$ B. Ruzicka, L. Zulian, and G. Ruocco, Langmuir 22, 1106 (2006).

${ }^{28}$ B. Berne and R. Pecora, Dynamic Light Scattering (Plenum, New York, 1985).

${ }^{29}$ See supplementary material at http://link.aps.org/supplemental/ 10.1103/PhysRevB.81.104201 for preliminary tests verifying the assumption that the rotational dynamics is much faster than the translational one, and tests the linearity of the response function.

${ }^{30}$ R. W. Boyd, Nonlinear Optics (Academic, San Diego, 2003).

${ }^{31}$ E. Hecht, Optics, 4th ed. (Addison-Wesley, Reading, 2001).

${ }^{32}$ Here $t_{w}=0$ is set as the time in which we stop stirring the prepared Laponite supension. Note that the first measurement of $T_{\text {eff }}$ is taken at $t_{w}=90 \mathrm{~min}$ but we expect $T_{\text {eff }}$ at $t_{w}=0$ to be quite similar to $T_{e f f}\left(t_{w}=90 \mathrm{~min}\right)$ since the correlation and response change extremely slowly at the beginning of the aging process. 